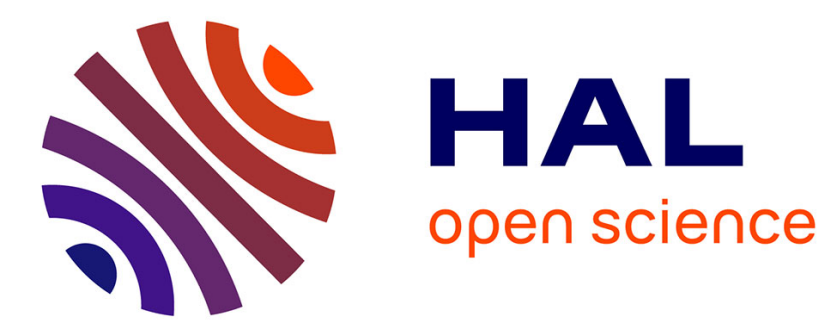

\title{
P2q hierarchical decomposition algorithm for quantile optimization: application to irrigation strategies
}

Olivier Crespo, Jacques-Eric J.-E. Bergez, Frederick Garcia

\section{To cite this version:}

Olivier Crespo, Jacques-Eric J.-E. Bergez, Frederick Garcia. P2q hierarchical decomposition algorithm for quantile optimization: application to irrigation strategies. Annals of Operations Research, 2011, 190 (1), pp.375-387. 10.1007/s10479-008-0503-2 . hal-00744319

\author{
HAL Id: hal-00744319 \\ https://hal.science/hal-00744319
}

Submitted on 22 Oct 2012

HAL is a multi-disciplinary open access archive for the deposit and dissemination of scientific research documents, whether they are published or not. The documents may come from teaching and research institutions in France or abroad, or from public or private research centers.
L'archive ouverte pluridisciplinaire HAL, est destinée au dépôt et à la diffusion de documents scientifiques de niveau recherche, publiés ou non, émanant des établissements d'enseignement et de recherche français ou étrangers, des laboratoires publics ou privés.

$$
\text { Copyright }
$$




\title{
$P 2 q$ hierarchical decomposition algorithm \\ for quantile optimization: application to irrigation strategies design
}

\author{
O. Crespo · J.É. Bergez - F. Garcia
}

Received: date / Accepted: date

\begin{abstract}
Decision theory dealing with uncertainty is usually considering criteria such as expected, minimum or maximum values. In economic areas, the quantile criterion is commonly used and provides significant advantages. This paper gives interest to the quantile optimization in decision making for designing irrigation strategies. We developed $P 2 q$, a hierarchical decomposition algorithm which belongs to the branching methods family. It consists in repeating the creation, evaluation and selection of smaller promising regions. Opposite to common approaches, the main criterion of interest is the $\alpha$-quantile where $\alpha$ is related to the decision maker risk acceptance. Results of an eight parameters optimization problem are presented. Quantile optimization provided optimal irrigation strategies that differed from thus reached with expected value optimization, responding more accurately to the decision maker preferences.
\end{abstract}

Keywords Simulation-based optimization · quantile optimization · irrigation management

\section{Introduction}

Quantiles are already largely used in many economic applications. For instance, the Value-at-Risk is defined as a quantile of the distribution of losses and is applied to

O. Crespo

CSAG, EnGeo Department, University of Cape Town,

Private Bag X3, Rondebosch 7701, Cape Town, South Africa

Tel.: +27 216503164 , Fax: +27 216505773

E-mail: olivier.crespo@csag.uct.ac.za

J.É. Bergez

INRA, UMR 1248 AGIR,

BP 52627, 31326 Castanet Tolosan, France

Tel.: +33561285 037, Fax: +33561 735537

E-mail: jbergez@toulouse.inra.fr

F. Garcia

INRA, Unité Biométrie et Intelligence Artificielle,

BP 52627, 31326 Castanet Tolosan, France

Tel.: +33 561285 283, Fax: +33 561285335

E-mail: fgarcia@toulouse.inra.fr 
risk management in finance, insurance, or banking (Jorion 2001). They are also largely involved in quantile regression (Koenker and Basset 1978) for optimal allocation design.

Rostek (2007) lists advantages of using the quantile instead of the mean or standard deviation when faced with a decision problem under uncertainty. The main ones are the improved robustness to fat tails and the predictions not driven by outliers. The improved robustness is due to the uselessness of responses quantification. The user does not need indeed to prefer one solution 10 times more than another, he just needs to rank them. This implies that no a priori preferences are needed, which allows to resolve the problem without user dependence. This conclusion is also true for maxmax and maxmin optimization. The former consists in maximizing the maximum payoff of a random variable, since the latter consists in maximizing the minimum one. The first kind of optimization is said optimistic, and the decision maker target is to increase the best expected outcome without regard to the worst cases. The second kind of optimization is said pessimistic, and the decision maker try to limit the worst achievable outcome by maximizing it. These criteria have been applied to game theory, robust control, bargaining and others areas. However these criteria have been criticized for basing the choice on what may be extreme and unlikely outcomes. Quantile optimization is then intermediate between the extremes maximum and minimum maximization. Robustness is still improved compared to mean and standard deviation analysis, and predictions are not driven by unlikely outliers. Even more, no user preferences are needed but a risk acceptance, called anticipation level in Rostek (2006).

The aim of this study is to design a simulation optimization algorithm adapted to quantiles optimization, and to analyze on some agricultural applications, the worth of optimizing quantiles instead of expected values. Due to the complex nature of our application, we assume that computing probability distributions or utility function is too expensive. We thus focus on optimization methods that do not need prior knowledge of the uncontrollable parameters. Simulation optimization concerns systems without an explicit analytical expression, but where instead only responses of sampled stochastic simulation runs $L$ are available. The formulation of that kind of optimization involves input variables $\theta$, constraints on these decision variables that should take value into a feasible region $\Theta$ and an objective function defined on these variables, $J: \theta \rightarrow \mathbb{R}$. The common formulation of the optimization problem consists in defining $J(\theta)$ as the expected value $E[L(\theta, \omega)]$ with $\omega$ the uncontrollable input variables vector of the stochastic system (Andradóttir (1998), Ólafsson and Kim (2002) Fu et al (2005)). Quantile optimization consists then in replacing the expected value of $L$ by the quantile of order $\alpha$ :

$$
\max _{\theta \in \Theta} J(\theta)=q_{\alpha}[L(\theta, \omega)] .
$$

We present in this paper $P 2 q$, a new and original algorithm for solving this difficult simulation optimization problem. We applied the $P 2 q$ algorithm to the problem of optimizing irrigation strategies for corn, based on the crop simulation model MODERATO (Bergez et al 2001) in order to get the greatest simulated yield. This case application is a good illustration of the kind of simulation optimization problems that exists in crop management.

The plan of the paper is the following: first, we define quantiles and give limitation to the quantile optimization problem. Next, we describe the principle of the $P 2 q$ algorithm we propose and the risk interpretation of the quantile of order $\alpha$. In section 4 , we give some information on the biodecisional simulation model used to simulate the irrigation strategies and we set up the simulation experiments. We then present the 


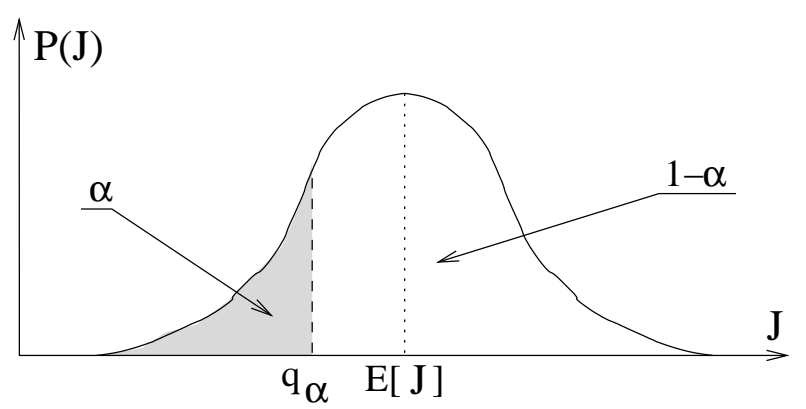

Fig. 1 Quantile of order $\alpha$ separating the probability distribution of a random variable.

results of the quantile optimization for different quantile orders, and we discuss the pros and cons of this quantile optimization approach.

\section{Quantile optimization}

As extremes pessimistic and optimistic indices can be optimized with maxmin and maxmax approaches, a non extreme risk acceptance index can be improved using quantile optimization. Furthermore in most of the cases, quantile estimation will rely on more possible outcomes than the unique extreme taken into account for either maxmax or maxmin optimizations. In opposition to the usual mean expected value provided to decision makers, the quantile estimation can provide relative pessimistic or optimistic options in accordance to decision risk acceptance. This new optimization point of view is a tool that can provide relevant advices with both farmers that want to increase the $X \%$ worst possible outcomes or those who focus on the $X \%$ best.

Let $\alpha$ be a fixed probability in ]0,1[. The quantile of order $\alpha$ of the random variable $J$ is the threshold $q_{\alpha}$ such that the probability of observing $J$ lower than $q_{\alpha}$ is equal to $\alpha: P\left(J<q_{\alpha}\right)=\alpha$. For instance, if we are interested in the $10 \%$ highest values of $J$, we will compute the quantile of order $\alpha=0.9$. If we take $\alpha=0.5$ then we get the median. Figure 1 shows the probability distribution of the random variable $J$. The quantile $q_{\alpha}$ of order $\alpha$ is dividing the surface into two parts. The left hand side represents the probability of reaching a value lower than $q_{\alpha}$ : it is equal to $\alpha$. The right hand side represents the probability of reaching a value greater than $q_{\alpha}$ : it is equal to $1-\alpha$.

The optimization problem we consider consists in optimizing the $\alpha$-quantile of the random variable $L(\theta, \omega)$. The decision maker has thus to characterize his risk acceptance through the order $\alpha$. This scalar $\alpha \in[0,1]$ is a subjective belief up to the decision maker, such that $\alpha=0$ is equivalent to a maxmin (pessimistic) approach and $\alpha=1$ is a maxmax (optimistic) approach.

The common objective function is transformed from the expected value of simulation outcomes into the quantile of order $\alpha$, which leads usually to different decisions. Thus the use of quantile in simulation-based optimization is not vain and we propose hereafter to evaluate its interest for irrigation strategies design. 
3 The $P 2 q$ algorithm

3.1 A hierarchical algorithm

The $P 2 q$ algorithm is an extension of the $P 2$ and $P 2 p$ algorithms we introduced in Crespo et al (2008) and Bergez et al (2004). It is based on a hierarchical decomposition of the decision space into a binary tree and belongs to the family of stochastic branching methods, like stochastic branch-and-bound or nested partitions methods. It is dedicated to large continuous input variables problems. It encloses a single objective function, which can be the combination of weighted multiple objectives.

The decision space $\Theta$ is a hyper-rectangle or region. The $P 2 q$ optimization aims at finding small hyper-rectangles that we call $R$ which include the decision vectors that maximize the quantile of order $\alpha$ of performance measures $L(\theta, \omega)$. We assume that a hyper-rectangle is small enough, or unbreakable, when any decision vector included in this hyper-rectangle is indiscernible from the others. This is defined by the user for every dimension $d$ of the $D$-dimensional problem as the width step of the dimension $d \in D$.

Let us call pending regions the regions that are still breakable (in opposition to the unbreakable ones). Initialization allocates the initial decision space as the single pending region of the list pending $R L$ of all the pending regions. The first step consists in SELECTING out from the pending regions list pending $R L$, the region which is potentially optimal: we call it the promising region. This promising region is either selected in accordance to one or two indices evaluated from the decision vectors included in the pending regions, or it is randomly chosen following a low probability set for the whole process. The second step DIVIDES this promising region into two parts either following a trivial equal partitioning process or techniques looking at producing the most different sub regions. These two parts are offspring regions and are collected in the offspring $R L$ list. During the third step, each of these offspring $R L$ regions is sampled uniformly, simulated and quantiles are EVALUATED. This evaluation is the main concern of this study. Eventually, the pending regions list pending $R L$ is updated, and the three previous steps are repeated until stopping criteria are completed (usually time and/or simulation runs limits) or the pending regions list is empty.

The three main steps of selection, division and evaluation are discussed in Crespo et al (2008). The combination included in the $P 2 q$ algorithm involves the three following techniques. Selection considers only the quantile of order $\alpha$ of the performance measures simulated for each pending region $R \in$ pending $R L$. The division technique involves the determination of one included vector which translates as best as possible an ideal (defined by the user) separator vector called pivot. The promising region is then cut apart along the previously defined pivot vector in order to split the longest dimension $d \in D$ into two parts. The quantile of performance measures is computed during the evaluation step as defined in the following section 3.2. 
3.2 Quantile evaluation

Let us call $R \subset \Theta$ a decision subspace that includes decision vectors $\theta$. The evaluation of $R$ relies on $N \times M$ performance measures $L\left(\theta_{i}, \omega_{j}\right)$ simulated $(i=[1 . . N], j=[1 . . M]$ ). The number $N$ of $\theta$ simulated is proportionally related to the widest dimension of the considered subspace. These $N$ decision vectors $\theta$ are uniformly sampled within the $R$ decision subspace. Each of these decision is simulated coupled with $M$ uncontrollable parameter $\omega$ randomly selected within a collection of either historical observed or computationally generated $\omega$. The amount $M$ of uncontrollable $\omega$ is set for the whole process.

Let $\alpha \in[0,1]$ be a scalar such that the quantile $q_{0}\left[L\left(\theta_{i}, \omega_{j}\right)\right]$ is the smallest value of the performance measures and $q_{1}\left[L\left(\theta_{i}, \omega_{j}\right)\right]$ the largest. We use a weighted average method to estimate the quantile of order $\alpha$. The first step consists in ordering the performance measures within a region such that $L_{0}$ is the smallest value and $L_{(N \times M)-1}$ is the largest. Let $p$ be the integer part of $((N \times M)-1) \times \alpha$, and let $g$ be its fractional part. The weighted average estimation of the $\alpha$ order quantile of performance measures is done as follow.

$$
q_{\alpha}\left[L\left(\theta_{i}, \omega_{j}\right)\right]=L_{p}+g \times\left(L_{p+1}-L_{p}\right)
$$

According to the previous quantile estimation process, the evaluation criterion $J(R)$ of the decision subspace $R$ is computed as follow.

$$
J(R)_{\theta \in R}=q_{\alpha}[L(\theta, \omega)] .
$$

\section{Application to irrigation management}

We applied this new approach to design of irrigation strategies. The aim of this section is to present the problem we used to test the $P 2 q$ algorithm.

\subsection{MODERATO Simulator}

MODERATO (Bergez et al 2001) is a plot-based annual-based model aimed at evaluating current irrigation strategies for corn and at proposing improved strategies. It has been developed in close collaboration with irrigation advisers. It combines a dynamic and biophysical corn crop model with a dynamic decision model. The crop model is described in Wallach et al (2001). In short, the model is based on the general captation / conversion of the solar radiation allowing for leaf area and biomass development. Depending on phenological stages, efficiency of radiation conversion differs. Yield is calculated thank to the use of a dynamic harvest index. A water budget integrating transpiration and evaporation is computed on a 4-layer soil. Water stress modifies plant growth and harvest index.

The decision model consists of a set of decision rules for different management decisions. A decision rule can be seen as a function linking some indicators of system status, in this case the cropping system, to the action to be performed. It is written as a Boolean condition: "IF < indicator $><$ operator $><$ threshold $>$ THEN < action1 $>$ ELSE < action2>". A crop management strategy is composed by three simple rules (Sowing, Fertilizing and Harvest) and by the Irrigation Strategy, which, in its turn, is defined by five elementary rules: $i$ ) a rule to determine if irrigation is to be used 
to facilitate plant emergence (Irrigation at Sowing Rule); ii) a rule to decide when to start the main irrigation period (Starting Rule); iii) a rule to determine when to start a new irrigation round (Returning Rule); $i v$ ) a rule to delay irrigation due to weather conditions (Delaying Rule); $v$ ) a rule to decide when to stop irrigation (Ending Rule). The crop model updates the state variables each day and passes their values to the decision model together with the explanatory variables of that day.

The constraints to irrigation and available in MODERATO may be divided into three categories:

Equipment constraints: maximum flow available due to pumping capacity per hectare; maximum and minimum amount of irrigation allowed.

Resource constraints: a limited total amount of water for irrigation and a decreased flow rate during the irrigation period.

Regulatory or human constraints: irrigation bans on some days of the week, farmers reluctance to work for all or part of certain days, etc.

Constraints may vary during the irrigation period. For example river flow may decrease in summer, reducing the pumping flow. Regulations may be issued to reduce water consumption because of water shortage, possibly by prohibiting irrigation on certain days. These modifications of the general context over the irrigation period can be taken into account in the irrigation scheduling. However, as MODERATO is used at a strategic level, those modifications have to be anticipated to be tested.

An irrigation strategy is then defined as the set of rules (indicators - thresholds) activated for a given irrigation context.

MODERATO can run over a climatic series but every year is independent.

\subsection{Case study}

Tests have been run on an eight-parameter strategy as follows.

The main irrigation period starts from $\mathbf{T} 1$ as soon as the soil water deficit reaches D1. An amount I1 is applied. Once an irrigation cycle ends, a new cycle starts when the soil water deficit reaches D2. An amount I2 is applied. For the irrigation cycle following T3, if the soil water deficit is greater than D3 before this irrigation cycle starts, a last irrigation cycle is performed; otherwise the irrigation campaign ends. An amount $\mathbf{I} 3$ is applied.

Other cultural operations are modeled with decision rules, such as the sowing or the harvest. Details of these rules are provided in the table 1. Soil properties and climatic series used are describing south west of France. The variation in rainfall during the two summer months as it ranges from 30 to $240 \mathrm{~mm}$, underlining the unpredictable nature of rainfall in the area.

The irrigation equipment used for the study allows a $3.5 \mathrm{~mm} /$ day flow rate. A 180 $\mathrm{mm}$ limitation of available water is applied. No flow rate restrictions during summer (except those due to the equipment) are imposed.

The objective function to be maximized is the $\alpha$-quantile of the direct margin (i.e. the gross margin minus specific costs for a given activity, here irrigation). The direct margin regarding irrigation can be written as a weighted sum of multiple criteria:

$$
L\left(\theta_{i}, \omega_{j}\right)=a\left(\theta_{i}, \omega_{j}\right) \cdot B-\left[C+d\left(\theta_{i}, \omega_{j}\right) \cdot E+f\left(\theta_{i}, \omega_{j}\right) \cdot G\right]
$$




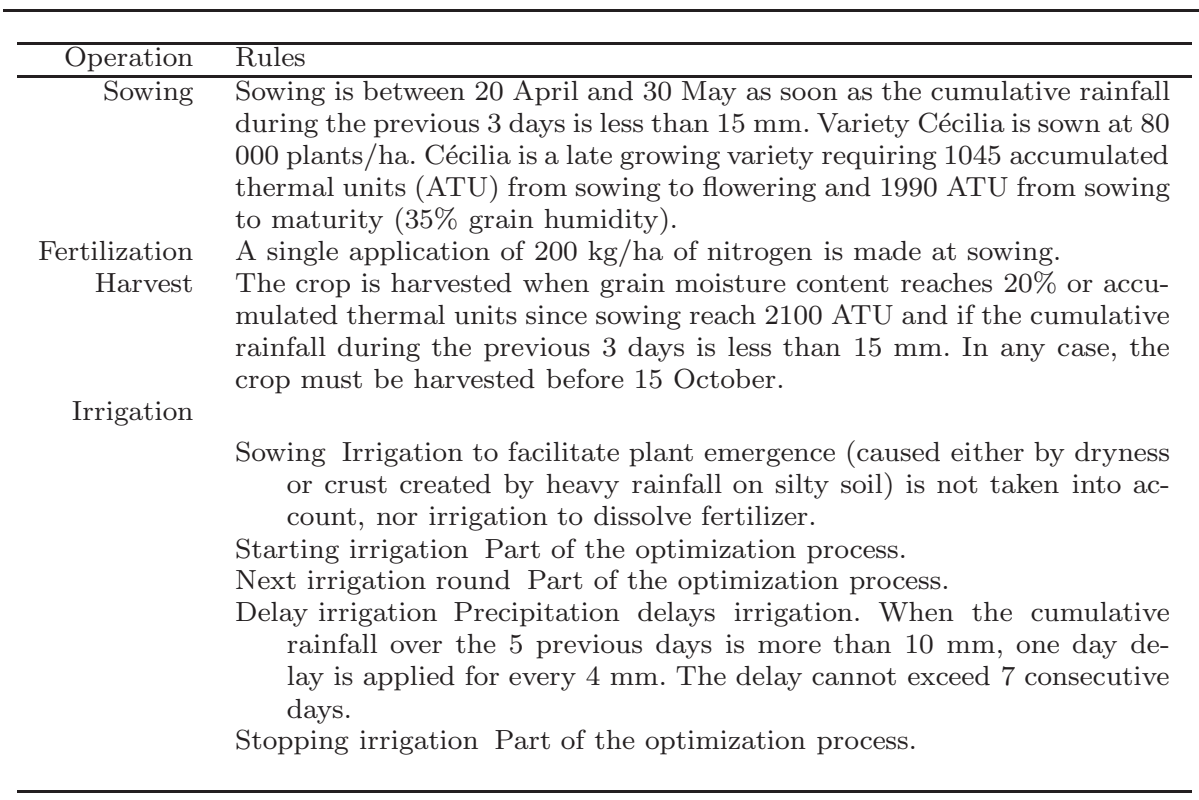

Table 1 General description of the strategy simulated.

where $L\left(\theta_{i}, \omega_{j}\right)$ is the direct margin for climate $\omega_{j}$ and the strategy $\theta_{i}, a\left(\theta_{i}, \omega_{j}\right)$ is the grain yield obtained under climate $\omega_{j}$ and using the strategy $\theta_{i}, B$ is the selling price for corn, $C$ is the operational costs for corn production, $d\left(\theta_{i}, \omega_{j}\right)$ is the amount of water used under climate $\omega_{j}$ and using the strategy $\theta_{i}, E$ is the cost of irrigation water, $f\left(\theta_{i}, \omega_{j}\right)$ is the number of irrigation cycles performed under climate $\omega_{j}$ and using the strategy $\theta_{i}$ and $G$ is the cost of carrying out a new irrigation cycle. The average selling price for maize (grain) is assumed to be $106.71 € / \mathrm{Mg}$ in the Toulouse area. Operational costs (seed, weeding, fertilizer, insurance) are assumed to be $327.77 € /$ ha. The cost of irrigation water is assumed to be $0.76 € / \mathrm{mm}$ and the setting up of a new irrigation cycle is assumed to be $7.62 € /$ ha.

\subsection{Experiments}

We considered 10 replications for each alternative. The initial feasible region is defined in table 2 as the ranges of the different parameters from the strategy described in section 4.2 .

Some procedure parameters were set for all experiments. The maximum number of simulation was set to 2 million. The probability of randomly choosing the promising region is set to $20 \%$ and the sampling follows a uniform distribution. The density of samples which are use to evaluate a decision subspace is set to 1 . According to the $P 2$ procedure, it means that the region $R$ evaluation is based on at least as many $\theta$ as step number along the widest dimension. Finally the number $M$ of uncontrollable $\omega$ is set to 49 and they are randomly chosen in weather data observed in the area of concern.

Each time the direct margin quantile increased, we stored it as well as the number of simulation runs used to achieve it. These stored values presented a strictly increasing 
Table 2 The eight parameters of the irrigation strategy to be optimized. Min and max show the range of each parameter within which the optimum is sought. A step is the minimum feasible range of the parameter.

\begin{tabular}{|c|c|c|c|c|c|}
\hline Names & Meaning & unit & $\min$ & $\max$ & step \\
\hline $\mathrm{T} 1$ & $\begin{array}{l}\text { Accumulated thermal unit to start the irriga- } \\
\text { tion campaign }\end{array}$ & ${ }^{\circ}$ C.day & 200 & 1250 & 5 \\
\hline D1 & Soil water deficit to start the irrigation & $\mathrm{mm}$ & 20 & 150 & 3 \\
\hline I1 & Irrigation applied at the first irrigation & $\mathrm{mm}$ & 5 & 50 & 2 \\
\hline D2 & Soil water deficit to start a new irrigation cycle & $\mathrm{mm}$ & 20 & 150 & 3 \\
\hline I2 & Irrigation depth applied & $\mathrm{mm}$ & 5 & 50 & 2 \\
\hline T3 & $\begin{array}{l}\text { Accumulated thermal units to stop the irriga- } \\
\text { tion }\end{array}$ & ${ }^{\circ} \mathrm{C}$.day & 1250 & 2000 & 5 \\
\hline D3 & Soil water deficit to stop irrigation & $\mathrm{mm}$ & 20 & 150 & 3 \\
\hline I3 & Irrigation applied at the last irrigation round & $\mathrm{mm}$ & 5 & 50 & 2 \\
\hline
\end{tabular}

curve in the simulation runs/direct margin criteria space. The general shape of this curve was made up of two distinct phases: one with a large improvement in direct margin quantile with a few simulation runs, and another with a tiny improvement with an infinite number of simulation runs. The large amount of simulation runs insured that every optimization processes reached the second phase of low improvement.

In order to highlight the advantages of quantile optimization, we first compare optimal regions reached for expected value and quantile optimizations. Then we compared quantile optimization of order 0.1 to order 0.9 . The optimization process included in $P 2 q$ is a minimization. The translation of any maximization into a minimization allows any problem to be handled. However in this case, in opposition to what we said in the earlier quantile definition section, the quantile of order 0.1 divided the $10 \%$ best solutions from the $90 \%$ worst, and the quantile of order 0.9 divided the $90 \%$ best from the $10 \%$ worst.

\section{Results}

5.1 Quantile vs. expected value optimizations

Figure 2 shows 50 random strategies simulated in four regions. The + are strategies included in the initial research domain. The $\times$ are strategies included in the optimal region reached with expected value optimization. The $*$ are strategies included in the optimal region reached with 0.1-quantile optimization. The $\square$ are strategies included in the optimal region reached with 0.9-quantile optimization. Every strategy is plotted two times. The first is upside and is located on the graph by its average value and its 0.1 -quantile. The second is downside and is located on the graph by its average value and its 0.9-quantile.

The optimal regions reached high $\alpha$-quantile and expected value comparing to the strategies simulated within all the decision space. In one hand, the 50 strategies simulated in the 0.1-quantile optimal region are maximizing the threshold dividing the $10 \%$ best responses and the $90 \%$ lower ones. It reflects an optimistic decision indicator focusing on optimizing the best responses. In the other hand, the 50 strategies simulated in the 0.9-quantile optimal region are maximizing the threshold dividing the $90 \%$ best 


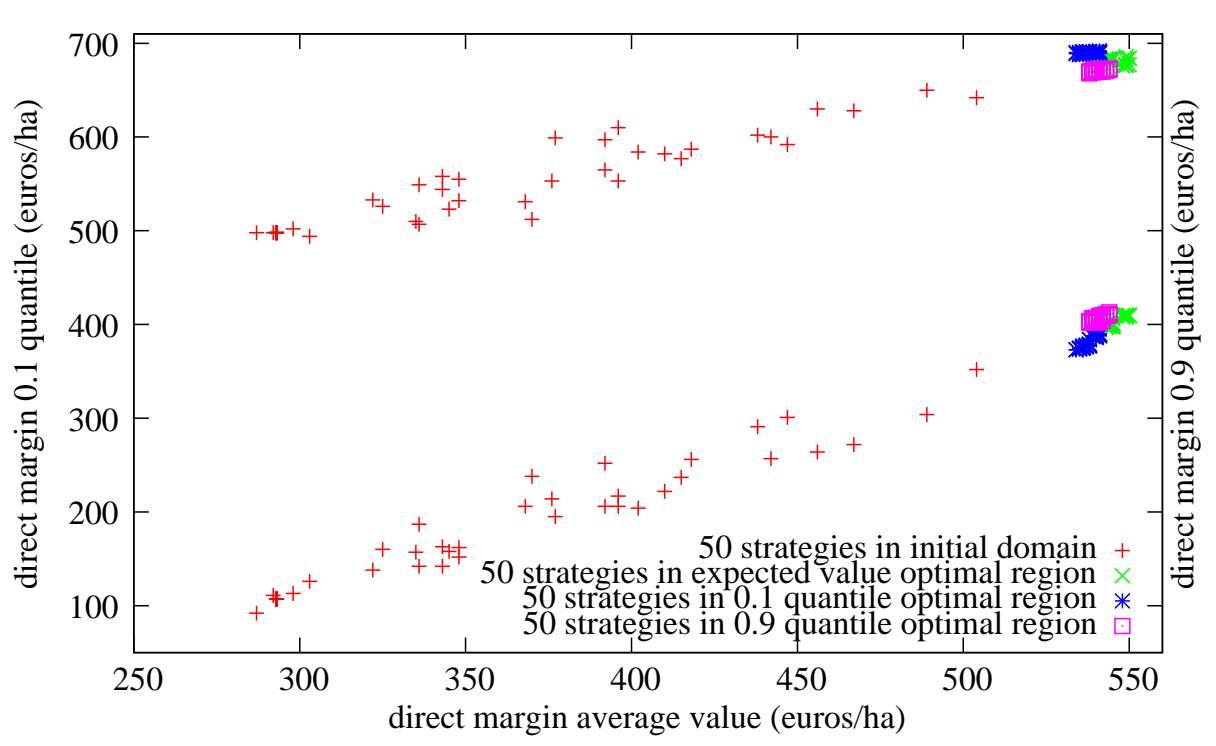

Fig. 2 Two times 50 strategies of four different regions.

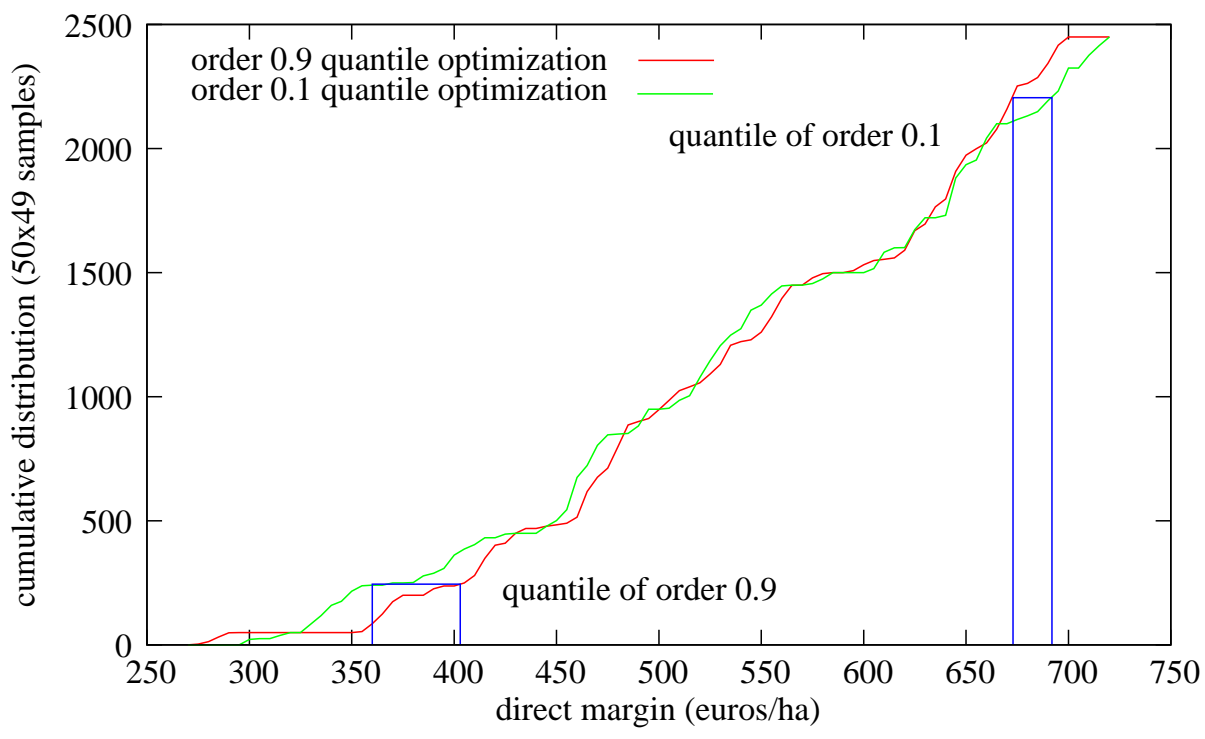

Fig. 3 Cumulative distributions based on $50 \times 49$ performance measures for each region.

responses and the $10 \%$ lower ones. It reflects a pessimistic decision indicator focusing on optimizing the worst responses.

\subsection{Different quantile orders optimizations}

Figure 3 shows the cumulative distributions of the optimal regions respectively reached with 0.1- and 0.9-quantile optimization. These distributions rely on 2450 performance 


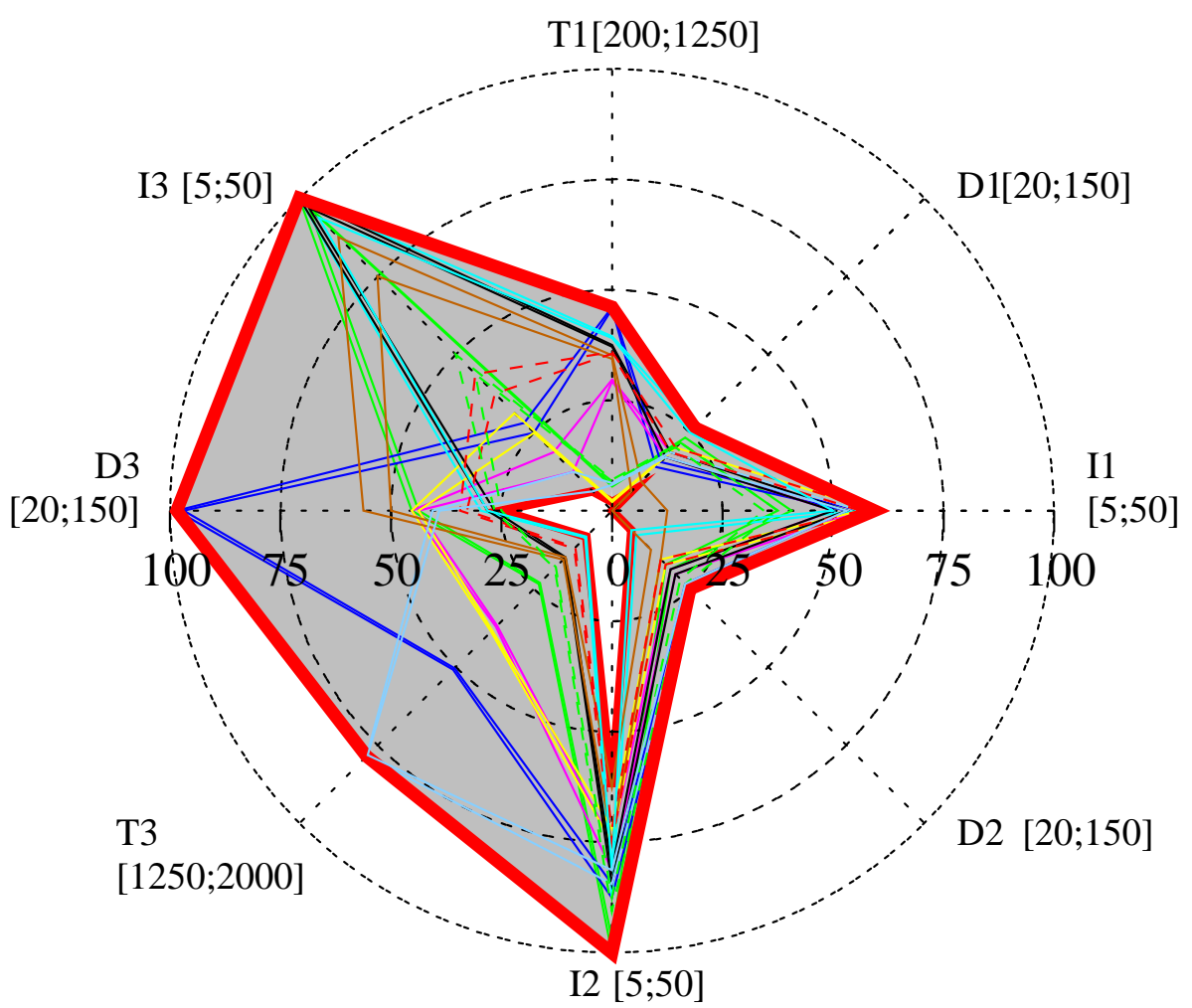

Fig. 4 Input variables envelope of the 10 optimal regions reached for the 10 replications of the $P 2 q$ algorithm: 0.1-quantile optimization.

measures, so that the 0.1-quantile divided the 245 best measures from the 2205 others and so that the 0.9-quantile divided the 245 worst measures from the 2205 others. These thresholds are pictured as $\sqcap$ upper bounded by 245 and 2205 measures from a distribution quantile to the other. So the width of these $\sqcap$ translates the improvement of the $\alpha$-quantile. For example, 0.9-quantile reached a value larger than $400 € / h a$ when the optimized quantile order is $\alpha=0.9$, and it reached a value close to $360 € / h a$ when the optimized quantile order is $\alpha=0.1$.

Latter figures characterized criteria responses of optimal regions. The next figures 4 and 5 are multiple axis charts representing the decision variable space. Couples of thin lines bound the parameters defining the optimal regions that were reached for the 10 replications of the $P 2 q$ algorithm. The couple of bold lines is an envelope of all of them. Each axis, leveled from 0 to 100, depicts each parameter from the minimum $(0 \%)$ to the maximum $(100 \%)$ values available and defined in table 2.

The largest 0.1-quantile direct margin reached over all replications is $691 € / h a$ and an average value of $537 € / h a$ (figures 3 and 4 ). The related decision vector depicted a first irrigation started after observing small accumulated temperature (below $725^{\circ} \mathrm{C}$.day) and water deficit (below $60 \mathrm{~mm}$ ), and the application of a medium amount of water (between 18 and $32 \mathrm{~mm} 8$ out of 10 times). The new irrigations are performed as soon as a small water deficit is observed (less than $55 \mathrm{~mm}$ ) and a large amount of water is applied (more than $32 \mathrm{~mm}$ ). The three last parameters leading to the eventual 


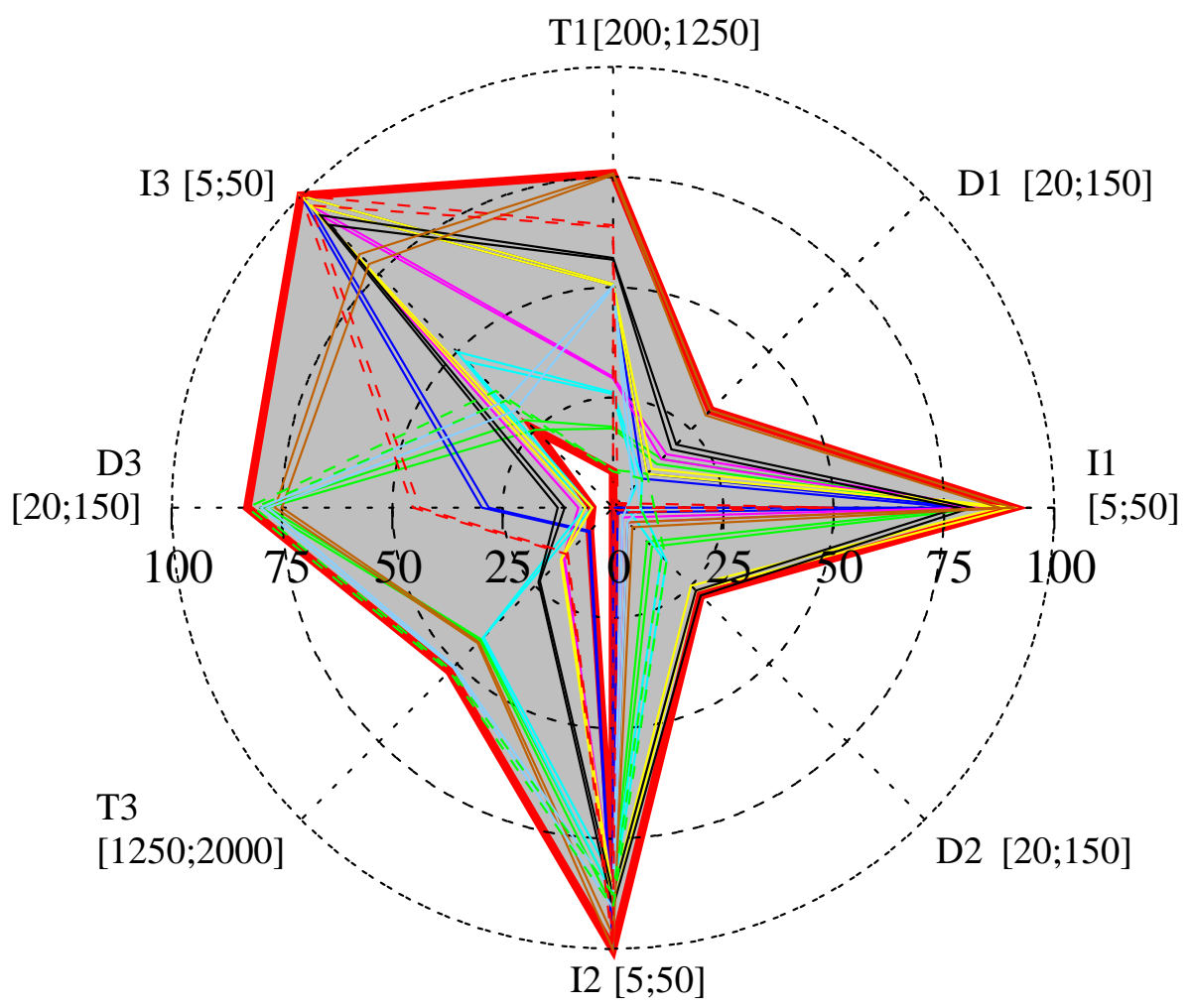

Fig. 5 Input variables envelope of the 10 optimal regions reached for the 10 replications of the $P 2 q$ algorithm: 0.9-quantile optimization.

irrigation campaign end are more variable. Yet we noticed that 6 out of 10 times the accumulated temperature $T 3$ leading to the irrigation campaign end is included in the $25 \%$ of low values (below $1440{ }^{\circ}$ C.day). In the meanwhile 8 out of 10 times the water deficit $D 3$ is included within the 25 and $50 \%$ of the proposed values (respectively 52 and $85 \mathrm{~mm}$ ). The last irrigation amount does not show any of the previous consistencies.

The largest 0.9-quantile direct margin reached over all replications is $412 € / h a$ and an average value of $543 € / h a$ (figures 3 and 5 ). The related decision vector depicted a first irrigation started after observing a wide accumulated temperature (from 200 to $1000{ }^{\circ}$ C.day) and small water deficit (below $40 \mathrm{~mm}$ ), and the application of a variable amount of water. The new irrigations are performed as soon as a small water deficit is observed (less than $40 \mathrm{~mm}$ ) and a large amount of water is applied (more than $32 \mathrm{~mm}$ ). The accumulated temperature $T 3$, the water deficit $D 3$ and the water amount I3 leading to the last irrigation are very variable and no consistency is ostensible. 


\section{Discussion}

6.1 Quantile optimization efficiency

As we expected, $P 2 q$ as $P 2$, reached optimal regions with high objective function average value. The highest direct margin average value was achieved with the usual expected value optimization of the performance measures. However, $\alpha$-quantile optimizations reached regions with greater $\alpha$-quantiles (figure 2).

This means that the region distribution shapes were distinct. Figure 3 shows the cumulative distributions of the optimal regions reached with 0.1 - and 0.9-quantile optimization. The deviation around the expected value which is about $540 € / h a$ in both cases, is very large. This highlights the significance of using quantile optimization. Indeed, optimization of the single expected value does not care about the shape of the region distribution. So, the decision maker can either reach optimal region with expected value probability of appearance close to one or zero. The perspective that the predicted decision will lead indifferently to the most likely or the most unlikely solution of optimal expected value, is not attractive. The optimization of quantile associated to the decision maker risk acceptance focus on more representative strategies and reduce the former misbehavior risk of appearance.

The results showed that the algorithm is as efficient to produce optimal region as the prior $P 2$. Furthermore it focuses on optimizing quantile thresholds which translate a new decision maker preference. Thus since the decision maker is able to characterize his risk acceptance, quantile optimization handled both with the efficiency of the predicted decision and the probability of its quantile of interest. That makes the quantile optimization more accurate to propose optimal decisions leading to optimal solutions according to the risk acceptance or aversion of the decision maker.

\subsection{Optimal irrigation plans}

Granting a large enough number of simulations, sensitive parameters are the same (figures 4 and 5). In one hand, the envelopes of optimal variables D1, D2 and I2 were thin, which underlines the large influence of these parameters. In the other hand, envelops of optimal variables $D 3$ and $I 3$ were wide, which underlines the small influence of these parameters. This observation was already true for the optimal decision space proposed with optimization of the expected value (figure 6). Main differences were related to $T 1$ and $I 1$ parameters. The 0.1 -quantile optimization led to relatively small intervals compared to the ones reached with 0.9-quantile optimization. We noticed two main limitations. The first one is that we simulated a water limited case, so that depending on the climate, the limit could have been reached or not and implied larger variability in the irrigation stopping rule decision parameters. The second one is that the decision parameter $I 2$ was always high valued and may have been larger depending on the range we set to it. But $I 2$ is an irrigation amount and its range variation should be discussed before any change.

Although the shapes of optimal regions were similar, the surface of the envelopes reached with quantile optimizations was larger than the one reached with expected value optimization. This observation corroborates that the quantile optimization is harder than expected value optimization. 


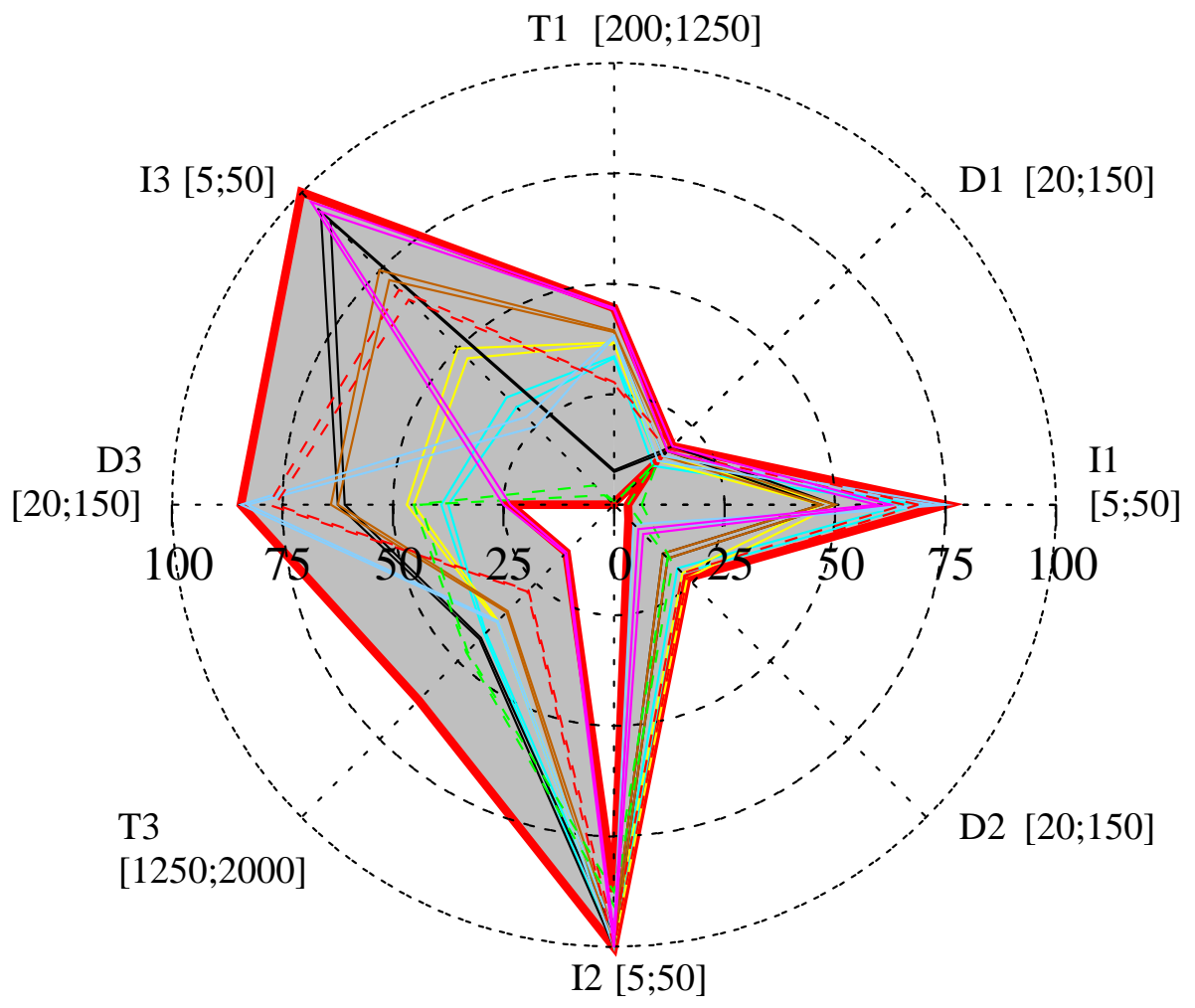

Fig. 6 Input variables envelope of the 10 optimal regions reached for the 10 replications of the $P 2$ algorithm: expected value optimization.

\section{Conclusion and perspectives}

Granting that the decision maker is able to characterize his risk acceptance, results showed that optimizing quantiles leads to different optimal regions than optimizing expected value. As quantiles optimization allow the decision maker to take into account a new preference (the risk acceptance) which is not involved in expected value optimization, the new optimal regions reached by the $P 2 q$ algorithm address more accurately the decision maker preferences. Although we noticed that quantile optimization requires an extra sorting process and thus is more time demanding, it adds significant value to the design of irrigation strategies.

Though quantile optimization is commonly used in economic fields, it is unusual to use it to design agricultural strategies. This study emphasized the usefulness of quantile as an optimization criterion. This value should even be increased if combined with multiobjective optimization procedures. It seems indeed very attractive to be able to maximize simultaneously a small and a large quantile orders. It means that we could maximize the yield of poor crops and maximize the yield of efficient crops at the same time, such that the algorithm reaches decision subspace that avoid the small pay-off while maximizing the large ones. 


\section{References}

Andradóttir S (1998) A review of simulation optimization techniques. In: Proccedings of the 1998 Winter Simulation Conference, pp 151-158

Bergez JE, Debaeke P, Deumier J, Lacroix B, Leenhardt D, Leroy P, Wallach D (2001) Moderato: an object-oriented decision tool for designing maize irrigation schedules. Ecological Modelling 137:43-60

Bergez JE, Garcia F, Lapasse L (2004) A hierarchical partitioning method for optimizing irrigation strategies. Agricultural Systems 80:235-253

Crespo O, Bergez JE, Garcia F (2008) P2 hierarchical decomposition procedure: application to irrigation strategies design. Submitted

Fu M, Glover F, April J (2005) Simulation Optimization: A Review, New Developments, and Applications. In: Winter Simulation Conference, 2005 Proceedings of the, pp 83-95

Jorion P (2001) Value at risk. McGraw-Hill New York

Koenker R, Basset G (1978) Quantile regression. Econometrica 46:33-50

Ólafsson S, Kim J (2002) Simulation optimization. In: Winter Proccedings of the 2002 Winter Simulation Conference, pp 79-84

Rostek M (2006) Reasoning in Strategic and Non-Strategic Interactions. PhD thesis, Yale University

Rostek M (2007) Quantile Maximization in Decision Theory, unpublished Manuscript

Wallach D, Goffinet B, Bergez J, Debaeke P, Leenhardt D, Aubertot J (2001) Parameter estimation for crop models a new approach and application to a corn model. Agronomy Journal 93(4):757-766 